ARTICLE

Received 1 Feb 2017 | Accepted 2 Mar 2017 | Published 11 May 2017

DOI: $10.1038 /$ ncomms15152

OPEN

\title{
Structure and interstitial iodide migration in hybrid perovskite methylammonium lead iodide
}

\author{
J.L. Minns' ${ }^{1}$ P. Zajdel ${ }^{2}$, D. Chernyshov ${ }^{3}$, W. van Beek ${ }^{3} \&$ M.A. Green ${ }^{1}$
}

Hybrid perovskites form an emerging family of exceptional light harvesting compounds. However, the mechanism underpinning their photovoltaic effect is still far from understood, which is impeded by a lack of clarity on their structures. Here we show that iodide ions in the methylammonium lead iodide migrate via interstitial sites at temperatures above $280 \mathrm{~K}$. This coincides with temperature dependent static distortions resulting in pseudocubic local symmetry. Based on bond distance analysis, the migrating and distorted iodines are at lengths consistent with the formation of $\mathrm{I}_{2}$ molecules, suggesting a $2 \mathrm{I}^{-} \rightarrow \mathrm{I}_{2}+2 e^{-}$redox couple. The actual formula of this compound is thus $\left(\mathrm{CH}_{3} \mathrm{NH}_{3}\right) \mathrm{Pbl}_{3-2 x}\left(\mathrm{I}_{2}\right)_{x}$ where $x \sim 0.007$ at room temperature. A crucial feature of the tetragonal structure is that the methylammonium ions do not sit centrally in the A-site cavity, but disordered around two off-centre orientations that facilitate the interstitial ion migration via a gate opening mechanism.

\footnotetext{
${ }^{1}$ School of Physical Sciences, Ingram Building, University of Kent, Canterbury, Kent CT2 7NH, UK. ${ }^{2}$ Institute of Physics, University of Silesia, ul. Uniwersytecka 4, 40007 Katowice, Poland. ${ }^{3}$ Swiss-Norwegian Beam Lines, European Synchrotron Radiation Facility, Polygone Scientifique Louis Néel, 6, Rue Jules Horowitz, 38000 Grenoble, France. Correspondence and requests for materials should be addressed to M.A.G. (email: m.green@kent.ac.uk).
} 
P erovskite structures, with the general formula, $\mathrm{ABO}_{3}$, form one of the most important and commercially exploited family of solids. Hybrid perovskites that contain both organic and inorganic components are a subset, where the A site is composed of an organic cation, such as methylammonium (MA), within a post transition metal halide framework, such as lead iodide. They have emerged since 2009 (ref. 1) as simple, low cost solar cell materials, with power conversion efficiencies that are becoming competitive with silicon ${ }^{2-7}$. Methylammonium lead iodide (MAPbI) undergoes a number of structural phase transitions as a function of temperature, including an orthorhombic-tetragonal-cubic evolution that is common in perovskites. Ion mobility and the rotational dynamics of the non-isotropic MA ion adds further complexity to the structure. Mobility of all three ions, $\mathrm{Pb}, \mathrm{I}$ and MA, have been extensively studied $^{8-12}$, but, as yet, no definitive mechanism has emerged, although iodide ions have been shown to play a key role ${ }^{13}$. The complex structural features and intrinsic disorder explains the large number of anomalies in the literature as to the exact symmetry and structural parameters ${ }^{14}$, which is suggestive of localized symmetry variations that is prevalent in solid electrolytes.

We show that iodide ions migrate through an interstitial (I3) position. This migration is only possible through a correlated rearrangement of the MA ions. Furthermore, a substantial local static distortion of the $\mathrm{Pb}-\mathrm{I}$ octahedra into a pseudocubic arrangement produces I-I bond distances consistent with the formation neutral $\mathrm{I}_{2}$ defects that could effectively act as electron/hole pairs.

\section{Results}

$\mathrm{X}$-ray and neutron diffraction. The maximum entropy method (MEM) is an analysis technique that can be applied to diffraction data that generates density maps without prior knowledge of symmetry and unit cell content, and therefore unbiased towards any specific structural model. It can provide information on subtle local distortions even when this scattering is extremely weak compared to the bulk diffraction ${ }^{15-17}$.

To gain a deeper insight into the structure of MAPbI we have performed both powder neutron, single crystal X-ray diffraction, and powder synchrotron X-ray studies. Details of the structure determination strategy are given in the Supplementary Note 1. The structure was solved in $I 4 / m$ space group with lattice parameters of $a=8.8756(1) \AA$ and $c=12.6517(3) \AA$. The $I 4 / m$ symmetry is not an isomorphic subgroup of the high temperature cubic perovskite $P m \overline{3} m$ space group, so not a common perovskite symmetry ${ }^{18}$. However, in the case of $\mathrm{MAPbI}$ the transition from cubic to tetragonal is first order, so multiple irreducible representations can be adopted. The principal features of the MAPbI structure derived from the MEM analysis was found to be considerably more complex than previous realized (Fig. 1). Supplementary Fig. 1 shows the crystal structure from positions extracted from MEM analysis of powder neutron diffraction data (Supplementary Fig. 2). The nuclear scattering density around the iodide ion at the $(-0.2148(3)$ -0.2851 (3) 0.5 ) position (I2) at room temperature was found to be localized with typical levels of thermal distribution (Fig. 1a). However, additional densities with similarly localized scattering were identified at two positions in close proximity, demonstrating static disorder of the I2 site, labelled I2A. These were determined to be at $(-0.252(3)-0.248(3) 0.453)$ in a pseudocubic arrangement, and represent a $\sim 0.8 \AA$ shift from the known $\mathrm{I} 2$ position towards the $\mathrm{MA}$ ions and lying on either side of a mirror plane in the tetragonal space group. Supplementary Fig. 3 shows how the local tilting of the perovskite is different in I2A and $\mathrm{I} 2$ positions. The MEM density maps surrounding the MA ion for single crystal X-ray and powder neutron diffraction showed similar scattering for the $\mathrm{C}-\mathrm{N}$ that is best described as a 4 atom tetrahedron unit, with the centre of the tetrahedron in the middle of the A-site cavity (Fig. 1b,c, respectively). In addition to the scattering for $\mathrm{C} / \mathrm{N}$, the neutron diffraction data showed scattering from 6 hydrogen positions just over $1 \AA$ from the $\mathrm{C} / \mathrm{N}$ positions, with considerable amount of disorder through libration, as well as rotational disorder, which is consistent with inelastic neutron scattering results ${ }^{19,20}$. A model was derived for the MA ion (Fig. 1d), where there are two orientations for the $\mathrm{CH}_{3} \mathrm{NH}_{3}$ molecule, one in the (220) plane and one in the $(2 \overline{2} 0)$ plane, similar to the arrangement found from single crystal neutron diffraction ${ }^{21}$. These orientations point exactly between the iodide positions, I2, in the $z=0.25$ plane. However, whereas the centre of mass of the two molecule orientations lie also at $z=0.25$, the two molecules were found to be at off-centre positions of $z=0.221(3)$ and $0.279(3)$. The orientation of the MA molecule is an important component of the structure as it has been found to greatly influence the electronic properties of $\mathrm{MAPbI}^{22}$.

A further iodide position (I3) was observed in the powder neutron diffraction, powder synchrotron diffraction and single crystal X-ray diffraction that sits in an interstitial site in the $\mathrm{z} \sim 0.25$ plane with $\mathrm{Pb}$ and MA ions (Fig. 2a). Determination of the evolution of structure and composition as a function of temperature (Fig. 2b) showed a hysteresis effect in the heating and cooling profiles, demonstrating the phase transition is first order. Supplementary Fig. 4 shows the temperature dependence of specific reflection as measured from synchrotron radiation. The lattice parameters dependence showed a tetragonal to cubic transition at $\sim 335 \mathrm{~K}$, whereas this was suppressed to $\sim 320 \mathrm{~K}$ on cooling (Fig. 2b). This $15 \mathrm{~K}$ variation is mirrored in the composition variation (Fig. 2c) where the I1, I2 and I2A sites were similarly shifted. There was a slight drop in the composition of I1 sites close to the transition to the cubic phase, but the largest variation was in the occupancies of the I2 and I2A ions, where a substantial drop in $\mathrm{I} 2$ composition was observed with increases in I2A, but not to the same extent. The I3 content was difficult to accurately determine with the short runs of the synchrotron measurements. However, the total composition was seen to drop slightly, implying the I3 site were being populated, but were diffuse and thereby not contributing to the Bragg scattering to the same extent as the other iodide ions.

Ion migration mechanism. From the isolation of these atomic positions and variation of compositions, one can propose a mechanism for ion migration within the cell. The significant drop in composition of the I 2 site at temperatures above $280 \mathrm{~K}$, compared with the increased occupancy of the I2A site, implies that I2 are both populating the ion interstitial site, I3, and well as shifting to the new I2A locations. A I2 to I3 hop (Fig. 3a-c) would leave the $\mathrm{I} 3$ ions surrounded by three iodide ions all approximately $3.2 \AA$ apart. Given the concomitant increase in the population of the both the I2A and I 3 sites over the same temperature regime, the nature of the bonding between these two positions is important. Supplementary Fig. 5 shows the bond distances when all four iodide positions are populated. Polyiodide ions are well known to the form multiple low valent iodide chains, where the I-I bond lengths are extremely sensitive to the nature of the bonding and charges on the iodine ${ }^{23}$. Structure of solid $\mathrm{I}_{2}$ is an orthorhombic zig-zag structure with intramolecular I-I bond lengths of $2.68 \AA$, and intermolecular $\mathrm{I}_{2}$ distances of $3.56 \AA$ (ref. 24). This value of $2.68 \AA$ can be considered as a primary covalent I-I bond and other forms of iodine chains where $\mathrm{I}_{2}$ donates to the $\sigma^{\star}$ antibonding orbital in a charge transfer 

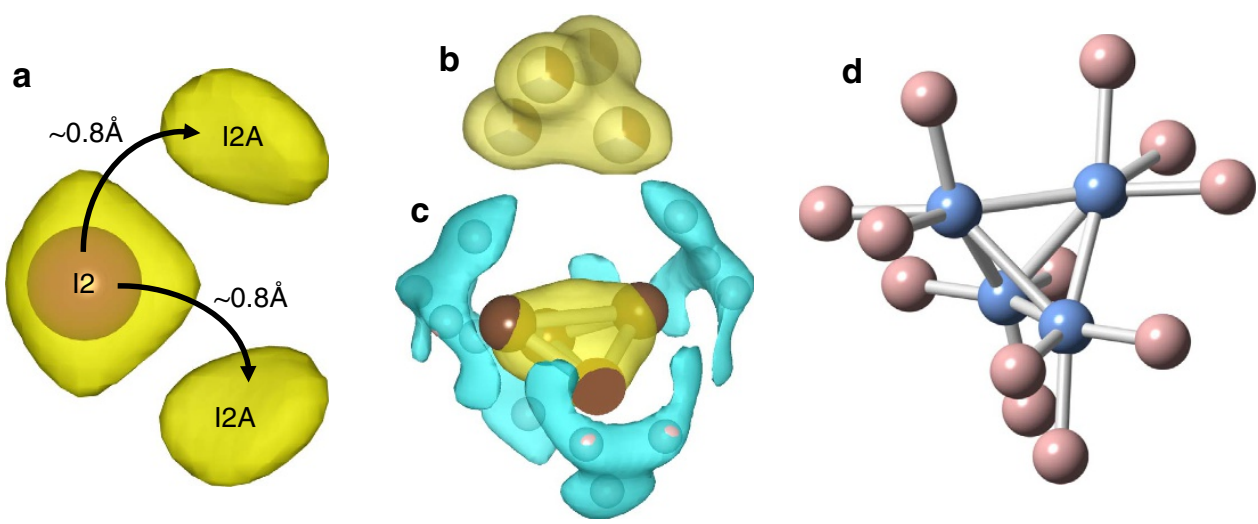

Figure 1 | Key structural features of MAPbl obtained from MEM analysis. (a) Section of the (100) projection of the nuclear scattering density (yellow) at room temperature (isosurface level of $0.8 \mathrm{fm} \AA^{-3}$ ) showing main iodide position (I2, purple sphere) is accompanied by two additional scattering densities (labelled I2A). (b) X-ray scattering and (c) nuclear scattering density map of methylammonium molecules (isosurface level of $1.0 \mathrm{fm} \AA-3$ ), showing $\mathrm{C}$ and $\mathrm{N}$ (yellow) and hydrogen scattering (blue) (d) molecular structure extracted from the maxima in the scattering density maps.
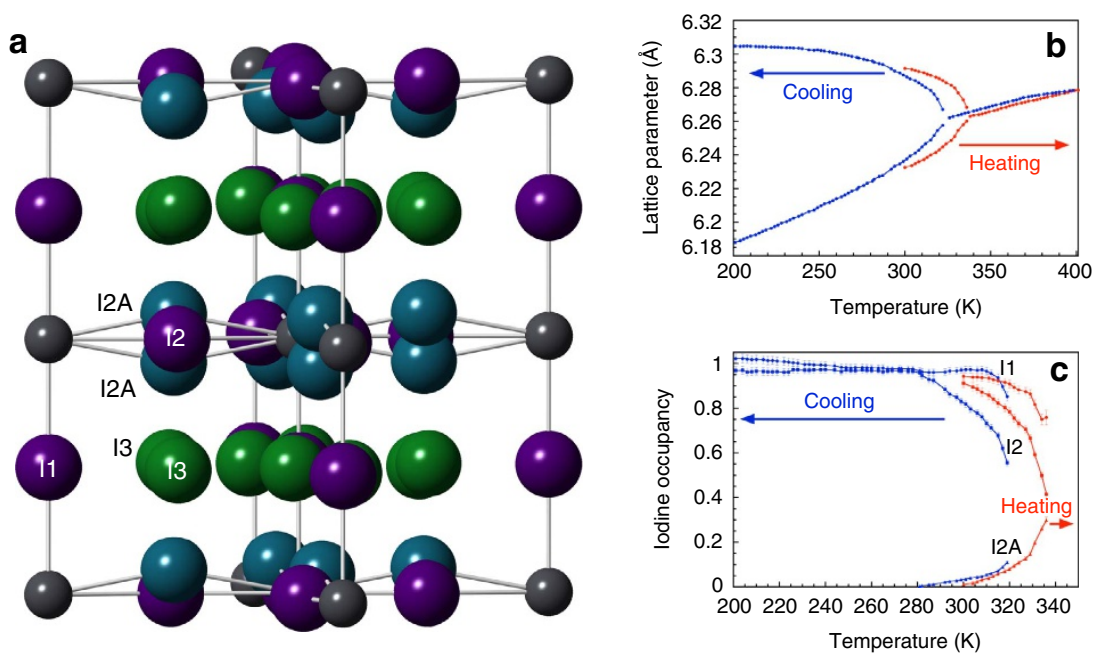

Figure 2 | Structure of MAPbl and variation of occupancy and lattice parameter with temperature. (a) Lead (grey spheres) and iodide positions in the room temperature $14 / \mathrm{m}$ space group, showing four crystallographically inequivalent iodide positions within the unit cell at position I1 and 12 (purple) that form the regular perovskite $\mathrm{Pbl}_{6}$ corner shared octahedra and two additional position I2A (blue sphere) and I3 (green sphere). The methylammonium ions are omitted for clarity. Powder synchrotron X-ray data shows (b) hysteresis in tetragonal to cubic phase transition, and (c) temperature dependence of the 11,12 and $12 \mathrm{~A}$ iodide ion site occupancy as a function of temperature.

complex have significantly larger bond lengths $\mathrm{s}^{23,25}$. For example, the triiodide ion, $\left[\mathrm{I}_{3}\right]^{-}$in orthorhombic $\mathrm{CsI}_{3}$ has $\mathrm{I}-\mathrm{I}$ bond lengths of $2.84 \AA$ and $3.04 \AA$ (ref. 26); one slightly higher than seen in covalent $I_{2}$ and one longer bond possessing most of the additional charge. $\mathrm{I}_{2}$ confined within frameworks have similar bond lengths, such as iodine in formate, $\mathrm{Zn}_{3}(\mathrm{HCOO})_{6}$, has a bond length of $2.691 \AA$ with a second weakly interacting molecule at $3.59 \AA$ (ref. 27). In contrast, the $\left[\mathrm{I}_{2}\right]^{+}$ion in $\mathrm{I}_{2} \mathrm{Sb}_{2} \mathrm{~F}_{11}$ has a shorter I-I bond length of just $2.56 \AA$ (ref. 28). The two I3-I2A bond lengths in MAPbI are at 2.7(1) $\AA$ and 2.6(1) $\AA$, so from these structural considerations it is consistent with the static disorder and shift from I2 to I2A is the result of covalent $\mathrm{I}_{2}$ bond formation in MAPbI to produce a neutral diatomic $\mathrm{I}_{2}$ molecule within the perovskite framework. This would have extensive implications on the band structure and charge transfer suggests a redox reaction of $2 \mathrm{I}^{-} \rightarrow \mathrm{I}_{2}+2 e^{-}$. The resulting structure (Fig. 4a) shows potential chains of $\mathrm{I}_{2}$ and $\mathrm{I}^{-}$ions along the $z$ axis. However, the bond distances between I3 and both orientations of the MA ions are unphysical, which suggests that the occupancy of I3 can only be achieved with the MA molecule adopting a perpendicular orientation (Fig. 4b), such that diffusion of the I3 ions from I2 and I1 only occurs with collective motion of the MA ions in a gate opening type mechanism. Further evidence of the dynamical hybrid structure was provided by Raman spectroscopy, which is shown in Supplementary Fig. 6, and Supplementary Note 2. All final derived atomic coordinates, occupancies are provided in Supplementary Tables 1-4.

Iodine, $\mathrm{I}_{2}$, itself is a semiconductor ${ }^{29}$, although can be highly conducting in polyiodide structures, or as inclusion in other frameworks creating charge transfer complexes ${ }^{23}$. A number of metal-organic frameworks materials have shown considerable change to their electronic structure and electrical conductivity with the inclusion of iodine ${ }^{30,31}$. Typical dye-sensitized solar cells require an additional $\mathrm{TiO}_{2}$ layer to act as an electron acceptor. However, it has been established that the hybrid perovskite itself acts as a free electron carrier without the need for complex nanostructures $3,5,32$, although the underlying mechanism for such characteristics is not clear. Further studies will be needed to clarify the presence and role of the $\mathrm{I}_{2} / \mathrm{I}^{-}$redox couple and the implied electron/hole formation within hybrid perovskite, as well 


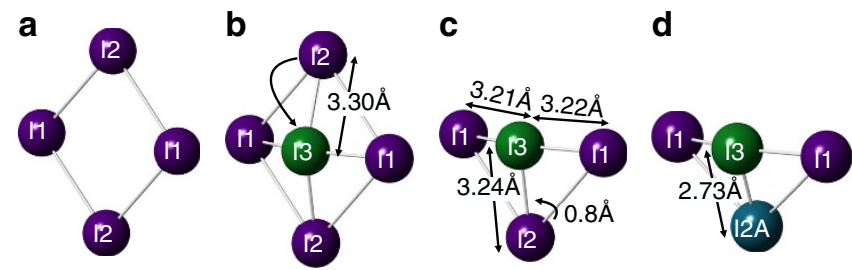

Figure 3 | Proposed mechanism for molecular iodine formation in MAPb (a) Section of the perovskite structure showing two 11 and two 12 position lying in the (110) plane, (b) lodine 12 moves to the interstitial 13 position leaving (c) 13 surrounded by three roughly equidistant iodine ions, provoking $\mathrm{I} 2$ ions to jump to a I2A position creating (d) bond formation to produce $\mathrm{I}_{2}$ molecules.
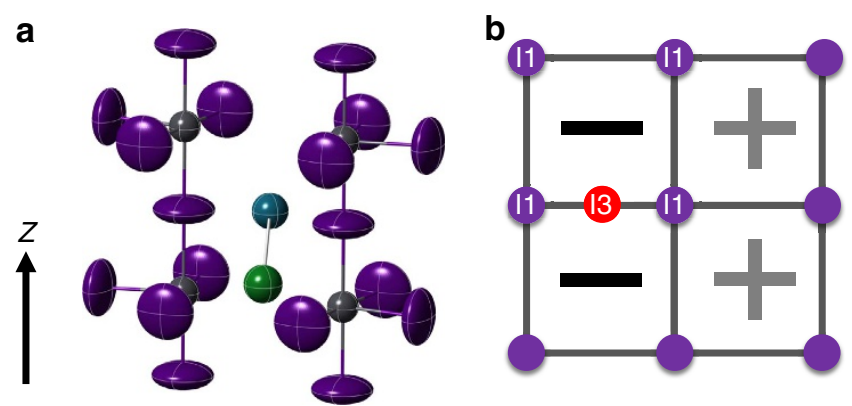

Figure 4 | Representative local structure of MAPbl after the proposed diatomic iodine formation. (a) Relative positions of $\mathrm{I}_{2}$ molecule (green and blue sphere) compared to the perovskite framework. Methylammonium ions are omitted for clarity, and (b) cooperative arrangements of the orientation of the methylammonium ions as a result of occupation of 13 sites. $(-)$ and $(+)$ represents single and shared orientations of MA molecule, respectively.

as the effect on the electronic and ionic conduction and whether this is related to its solar conversion properties, such as the long electron-hole diffusion lengths ${ }^{33}$ and lifetimes ${ }^{34}$, and photon recycling 35

\section{Methods}

Synthesis and data treatment. Crystals of MAPbI with a volume of $\sim 2 \mathrm{~cm}^{3}$ were grown by slow evaporation of $\mathrm{CH}_{3} \mathrm{NH}_{3} \mathrm{I}$ and $\mathrm{PbI}_{2}$ in $\gamma$-butyrolactone over a 14-day period. These crystals were ground to perform the powder neutron and X-ray studies, and smaller crystals were cleaved to perform the single crystal X-ray studies. Powder neutron diffraction data were collected on the BT1 diffractometer at NCNR at the National Institute of Standards and Technology, Gaithersburg, MD, USA using $\mathrm{Cu}(311)$ monochromator $(\lambda=1.5401(1) \AA)$. Single crystal X-ray diffraction data was collected on a dual-source Rigaku Oxford Diffraction Supernova diffractometer. Powder synchrotron diffraction experiments were performed at the Swiss-Norwegian beamline (SNBL) at the ESRF, France, with an incident wavelength of $\lambda=0.6932 \AA$. Whole pattern fitting based on MEM was carried out using PRIMA $^{36}$ and RIETAN ${ }^{37}$ with a $96 \times 96 \times 128$-voxel density map for both the powder neutron and single crystal X-ray diffraction data. MEM density maps were analysed using the Vesta program ${ }^{38}$. The FULLPROF ${ }^{39}$ suite of programs were used to perform Rietveld refinement on both the neutron and synchrotron X-ray powder diffraction data. Refinement of single crystal X-ray diffraction data were performed using the SHELX program ${ }^{40}$. Raman spectroscopy was performed with a Horiba LabRAM S3000 Raman microscope using a near infrared excitation wavelength of $784.15 \mathrm{~nm}$, and described in more detail in Supplementary Methods.

Data availability. The data that support the findings of this study are available from the corresponding authors upon reasonable request. The X-ray crystallographic coordinates for the structure reported in this study have been deposited at the Cambridge Crystallographic Data Centre (CCDC), under deposition number 1535723. These data can be obtained free of charge from The Cambridge Crystallographic Data Centre via www.ccdc.cam.ac.uk/data_request/cif.

\section{References}

1. Kojima, A., Teshima, K., Shirai, Y. \& Miyasaka, T. Organometal halide perovskites as visible-light sensitizers for photovoltaic cells. J. Am. Chem. Soc 131, 6050 (2009).

2. Im, J. H., Lee, C. R., Lee, J. W., Park, S. W. \& Park, N. G. 6.5\% efficient perovskite quantum-dot-sensitized solar cell. Nanoscale 3, 4088 (2011).

3. Lee, M. M., Teuscher, J., Miyasaka, T., Murakami, T. N. \& Snaith, H. J. Efficient hybrid solar cells based on meso-superstructured organometal halide perovskites. Science 338, 643 (2012).

4. Kim, H. S. et al. Lead iodide perovskite sensitized all-solid-state submicron thin film mesoscopic solar cell with efficiency exceeding 9\%. Sci. Rep. 2, 591 (2012).

5. Liu, M. Z., Johnston, M. B. \& Snaith, H. J. Efficient planar heterojunction perovskite solar cells by vapour deposition. Nature 501, 395 (2013).

6. Burschka, J. et al. Sequential deposition as a route to high-performance perovskite-sensitized solar cells. Nature 499, 316 (2013).

7. Jeon, N. J. et al. Compositional engineering of perovskite materials for high-performance solar cells. Nature 517, 476 (2015).

8. Yuan, Y. \& Huang, J. Ion migration in organometal trihalide perovskite and its impact on photovoltaic efficiency and stability. Acc. Chem. Res. 49, 286 (2016).

9. Eames, C. et al. Ionic transport in hybrid lead iodide perovskite solar cells. Nat. Commun. 6, 7497 (2015).

10. Dualeh, A. et al. Impedance spectroscopic analysis of lead iodide perovskitesensitized solid-state solar cells. ACS Nano 8, 362-373 (2014)

11. Walsh, A., Scanlon, D. O., Chen, S., Gong, X. G. \& Wei, S.-H. Self-regulation mechanism for charged point defects in hybrid halide perovskites. Angew. Chem. 54, 1791-1794 (2015).

12. Azpiroz, J. M., Mosconi, E., Bisquert, J. \& De Angelis, F. Defect migration in methylammonium lead iodide and its role in perovskite solar cell operation. Energy Environ. Sci. 8, 2118-2127 (2015).

13. Yang, T. Y., Gregori, G., Pellet, N., Gratzel, M. \& Maier, J. The significance of ion conduction in a hybrid organic-inorganic lead-iodide-based perovskite photosensitizer. Angew. Chem. 54, 7905 (2015).

14. Baikie, T. et al. Synthesis and crystal chemistry of the hybrid perovskite $\left(\mathrm{CH}_{3} \mathrm{NH}_{3}\right) \mathrm{PbI}_{3}$ for solid-state sensitised solar cell applications. J. Mater. Chem A 1, 5628 (2013)

15. Zajdel, P. et al. Phase separation and suppression of the structural and magnetic transitions in superconducting doped iron tellurides, $\mathrm{Fe}_{1+\mathrm{x}} \mathrm{Te}_{1-\mathrm{y}} \mathrm{S}_{\mathrm{y}} . \mathrm{J} . \mathrm{Am}$. Chem. Soc. 132, 13000 (2010).

16. Nakatsuji, S. et al. Spin-orbital short-range order on a honeycomb-based lattice. Science 336, 559 (2012).

17. Kuang, X. et al. Interstitial oxide ion conductivity in the layered tetrahedral network melilite structure. Nat. Mater. 7, 498 (2008).

18. Glazer, A. The classification of tilted octahedra in perovskites. Acta Crystallogr B 28, 3384 (1972).

19. Leguy, A. M. et al. The dynamics of methylammonium ions in hybrid organicinorganic perovskite solar cells. Nat. Commun. 6, 7124 (2015).

20. Chen, T. et al. Rotational dynamics of organic cations in the $\mathrm{CH}_{3} \mathrm{NH}_{3} \mathrm{PbI}_{3}$ perovskite. Phys. Chem. Chem. Phys. 17, 31278 (2015).

21. Ren, Y., Oswald, I. W. H., Wang, X., McCandless, G. T. \& Chan, J. Y. Orientation of organic cations in hybrid inorganic-organic perovskite $\mathrm{CH}_{3} \mathrm{NH}_{3} \mathrm{PbI}_{3}$ from subatomic resolution single crystal neutron diffraction structural studies. Cryst. Growth Des. 16, 2945 (2016).

22. Motta, C. et al. Revealing the role of organic cations in hybrid halide perovskite $\mathrm{CH}_{3} \mathrm{NH}_{3} \mathrm{PbI}_{3}$. Nat. Commun. 6, 7026 (2015).

23. Svensson, P. H. \& Kloo, L. Synthesis, structure, and bonding in polyiodide and metal iodide - iodine systems. Chem. Rev. 103, 1649 (2003).

24. Harris, P. M., Mack, E. \& Blake, F. C. The atomic arrangement in the crystal of orthrhombic iodine. J. Am. Chem. Soc. 50, 1583 (1928).

25. Alcock, N. W. in Advances in Inorganic Chemistry and Radiochemistry Vol. 15 (eds Emeléus, H. J. \& Sharpe, A. G.) 1-58 (Academic Press, 1972).

26. Tasman, H. A. \& Boswijk, K. H. Re-investigation of the crystal structure of $\mathrm{CsI}_{3}$ Acta Crystallogr. 8, 59 (1955)

27. Wang, Z. et al. $\left[\mathrm{Zn}_{3}(\mathrm{HCOO})_{6}\right]$ : a porous diamond framework conformable to guest inclusion. Aust. J. Chem. 59, 617 (2006).

28. Davies, C. G., Gillespie, R. J., Ireland, P. R. \& Sowa, J. M. The preparation and crystal structure of $\left[\mathrm{I}_{2}\right]^{+}\left[\mathrm{Sb}_{2} \mathrm{~F}_{11}\right]^{-}$. Can. J. Chem. 52, 2048 (1974).

29. Balchan, A. S. \& Drickamer, H. G. Effect of pressure on the resistance of iodine and selenium. J. Chem. Phys. 34, 1948-1949 (1961)

30. Zeng, M.-H. et al. Rigid pillars and double walls in a porous metal-organic framework: single-crystal to single-crystal, controlled uptake and release of iodine and electrical conductivity. J. Am. Chem. Soc. 132, 2561 (2010).

31. Zeng, M. H. et al. Nanoporous cobalt(II) MOF exhibiting four magnetic ground states and changes in gas sorption upon post-synthetic modification. J. Am Chem. Soc. 136, 4680 (2014). 
32. D’Innocenzo, V. et al. Excitons versus free charges in organo-lead tri-halide perovskites. Nat. Commun. 5, 3586 (2014).

33. Stranks, S. D. et al. Electron-hole diffusion lengths exceeding 1 micrometer in an organometal trihalide perovskite absorber. Science 342, 341-344 (2013).

34. Wehrenfennig, C., Eperon, G. E., Johnston, M. B., Snaith, H. J. \& Herz, L. M. High charge carrier mobilities and lifetimes in organolead trihalide perovskites. Adv. Mater. 26, 1584 (2014).

35. Pazos-Outón, L. M. et al. Photon recycling in lead iodide perovskite solar cells. Science 351, 1430 (2016).

36. Izumi, F. \& Dilanian, R. A. in Recent Research Developments in Physics, Vol. 3, 699 (Transworld Research Network, 2002).

37. Izumi, F. \& Ikeda, T. A rietveld-analysis programm RIETAN-98 and its applications to zeolites. Mater. Sci. Forum 321-324, 198-205 (2000).

38. Momma, K. \& Izumi, F. VESTA 3 for three-dimensional visualization of crystal, volumetric and morphology data. J. Appl. Crystallogr. 44, 1272 (2011).

39. Rodriguez-Carvajal, J. Recent advances in magnetic structure determination by neutron powder diffraction. Phys. B 192, 55 (1993).

40. Sheldrick, G. M. A short history of SHELX. Acta Crystallogr. A64, 112-122 (2008).

\section{Acknowledgements}

We thank the National Institute of Standards and Technology and Swiss-Norwegian beam line at the European Synchrotron Radiation Facility for provision of beam time. We thank the Royal Society for funding. We thank Dr H. Shepherd and P. Saines for assistance in performing the single crystal X-ray diffraction study.

\section{Author contributions}

M.A.G. synthesized the samples and designed the study. D.C. and W.v.B. performed the synchrotron measurements. M.A.G. performed the neutron experiments. J.L.M., P.Z. and
M.A.G. performed the analysis of the synchrotron, neutron and single crystal diffraction experiments. All authors contributed to the paper.

\section{Additional information}

Supplementary Information accompanies this paper at http://www.nature.com/ naturecommunications

Competing interests: The authors declare no competing financial interests.

Reprints and permission information is available online at http://npg.nature.com/ reprintsandpermissions/

How to cite this article: Minns, J. L. et al. Structure and interstitial iodide migration in hybrid perovskite methylammonium lead iodide. Nat. Commun. 8, 15152 doi: $10.1038 /$ ncomms15152 (2017).

Publisher's note: Springer Nature remains neutral with regard to jurisdictional claims in published maps and institutional affiliations.

(c) (i) This work is licensed under a Creative Commons Attribution 4.0 International License. The images or other third party material in this article are included in the article's Creative Commons license, unless indicated otherwise in the credit line; if the material is not included under the Creative Commons license, users will need to obtain permission from the license holder to reproduce the material. To view a copy of this license, visit http://creativecommons.org/licenses/by/4.0/

(C) The Author(s) 2017 